\title{
Optimal Solution Analysis and Decentralized Mechanisms for Peer-to-Peer Energy Markets
}

\author{
Dinh Hoa Nguyen *
}

\begin{abstract}
This paper studies the optimal clearing problem for prosumers in peer-to-peer $(\mathrm{P} 2 \mathrm{P})$ energy markets. It is proved that if no trade weights are enforced and the communication structure between successfully traded peers is connected, then the optimal clearing price and total traded powers in $\mathrm{P} 2 \mathrm{P}$ market are the same with that in the pool-based market. However, if such communication structure is unconnected, then the $\mathrm{P} 2 \mathrm{P}$ market is clustered into smaller P2P markets. If the trade weights are imposed, then the derived $\mathrm{P} 2 \mathrm{P}$ market solutions can be significantly changed. Next, a novel decentralized optimization approach is proposed to derive a trading mechanism for $\mathrm{P} 2 \mathrm{P}$ markets, based on the alternating direction method of multipliers (ADMM) which naturally fits into the bidirectional trading in P2P energy systems and converges reasonably fast. Analytical formulas of variable updates reveal insightful relations for each pair of prosumers on their individually traded prices and powers with their total traded powers. Further, based on those formulas, decentralized learning schemes for tuning parameters of prosumers cost functions are proposed to attain successful trading with total traded power amount as desired. Case studies on a synthetic system and the IEEE European Low Voltage Test Feeder are then carried out to verify the proposed approaches.

Keywords. Peer-to-Peer Energy Systems, Bilateral Trading, Optimal Energy Management, Multi-

${ }^{*}$ Dinh Hoa Nguyen is with the International Institute for Carbon-Neutral Energy Research (WPI-I ${ }^{2} \mathrm{CNER}$ ), and the Institute of Mathematics for Industry (IMI), Kyushu University, Fukuoka 819-0395, Japan. E-mail: hoa.nd@i2cner.kyushuu.ac.jp.
\end{abstract}

Agent System, ADMM, Decentralized Optimization.

\section{Nomenclature}

MAS Multi-agent system.

ICT Information and communication technology.

P2P Peer to peer.

DER Distributed energy resource.

ADMM Alternating direction method of multipliers.

$P_{i j}, P_{i}, P_{i, t r}$ Traded power between peers $i$ and $j$, vector of peer $i$ traded powers, and peer $i$ total traded power $[\mathrm{kW}]$.

$P_{i, t r}^{\min }, P_{i, t r}^{\max } \quad$ Lower and upper bounds of total traded amount of peer $i[\mathrm{~kW}]$.

$\mathcal{G}, \mathcal{A}, \mathcal{D}, \mathcal{L} \quad \mathrm{P} 2 \mathrm{P}$ interconnection graph, its adjacency, degree, and Laplacian matrices.

$\mathbf{1}_{n}, I_{n} \quad$ Vector with $n$ elements equal to 1 , and $n \times n$ identity matrix.

$\operatorname{diag}\{\}, \operatorname{vec}()$ Diagonal or block-diagonal matrices, and stacked vector.

$\mathbb{R}, \mathbb{R}^{n}, \mathbb{R}^{n \times m}$ Set of real numbers, real $n$-dimension vectors, and real matrices with dimensions $n \times m$. 


\section{Introduction}

P2P energy system has recently emerged as an attracting concept for novel energy market designs to push the flexibility, diversity, locality, and low emission of energy supply and consumption, due to the increasing penetration of DERs into energy grids 2, 24, 26, 27. First, energy losses are reduced in P2P systems because energy is usually exchanged within short distances. As a result, investment cost could be lower. Second, P2P trading platforms are often decentralized and localized, which are very suitable for integrating DERs and give much more flexibility for prosumers to handle their energy balance and profit. Next, equipped with advanced ICT technologies, e.g. distributed ledger technologies and block-chain, the security and privacy in P2P markets are much better than that in the conventional bulk energy grids [27. Last but not least, P2P trading helps promote new businesses since different models and market scales can be performed under P2P platforms, e.g. federated plans [17, full $\mathrm{P} 2 \mathrm{P}$, community-based, or their hybrid combination [16, 24. To this end, P2P energy system will serve as an important base to transform the current top-down, centralized energy networks into bottom-up, decentralized ones.

Each peer in P2P energy systems is a prosumer who can act as a producer at one time and as a consumer at another time. The trading of prosumers is affected by their preferences, e.g. energy transfer distance, or the sources of generated powers, etc. Usually, successful energy transactions are derived with short transfer distances and clean energy sources to avoid energy losses and reduce emissions of pollutants. Moreover, the role of each prosumer as a buyer (consumer) or seller (producer) can change from a time step to another, because its generation or consumption profile is time-varying and is only known by prediction in advance. Hence, the optimization problems arising from the optimal energy management or optimal planning and operation of $\mathrm{P} 2 \mathrm{P}$ energy systems are essentially different from that in pool-based energy markets.

Another feature distinguishing P2P energy market with other energy markets is on the direct energy trading between each prosumer (peer) with another communicated prosumer (peer). Therefore, a power balance constraint is forced to each pair of communicated prosumers, instead of only one balance constraint for the total generated and consumed powers of all producers and consumers (e.g. in poolbased markets). In order to deal with this pairing constraint, a number of different $\mathrm{P} 2 \mathrm{P}$ trading schemes has been proposed, e.g. bilateral contracts 4, 15, 19, 23, game theory based 6, 16, 25, 27, distribution optimal power flow 9, supply-demand ratio based pricing [13, mixed performance indexes [28], Lyapunov optimization [12, multi-class energy management [8, continuous double auction [9], etc.

To solve the optimal energy management problems arising in $\mathrm{P} 2 \mathrm{P}$ energy systems, decentralized optimization approaches are preferred to centralized or distributed optimization methods, because of the following reasons. First, decentralized approaches naturally fit into the structure of P2P energy systems, where no central coordination unit is needed and each peer directly communicates with other peers. Second, system robustness with respect to failure of individual parts is better with decentralized optimization approaches, because if some parts malfunctions, then the remaining still works, while centralized or distributed optimization methods have a single point of failure which stops the whole system from working if the central unit fails. Third, communication load is burdened at the central unit in centralized methods, but is much lessen at each node in decentralized approaches. As such, most of the existing optimization algorithms for P2P energy systems hitherto are decentralized.

ADMM is originally a distributed optimization approach which has been most often used in $\mathrm{P} 2 \mathrm{P}$ energy systems research [3, 4, 18, 29]. A direct application of ADMM to allocate exogenous costs in P2P energy systems was presented in 3], however local ADMM variables at each prosumer need to be updated in order. A distributed ADMM method together with model predictive control were introduced in [18] for solving a multi-class energy management problem, but a P2P platform agent exists for calculating dual prices and solving an optimal power flow problem. Coordination of $\mathrm{P} 2 \mathrm{P}$ energy trade and ancillary services was investigated in 29, where global variables 
on energy transfer matrix was required in the utilized ADMM algorithm. The effects of inter-peer communication sparsity to an ADMM algorithm convergence speed and market outcomes and the comparison between P2P market and other markets were studied in [3]. However, 3] considered the full communication structure which incurs more communication loads in P2P energy systems.

Comparison of different optimization approaches including ADMM with a central coordinator, primaldual algorithms, and Relaxed Consensus + Innovation (RCI) 23] for P2P energy systems has been conducted in 15. It turned out that the first method is fastest, while the last method is slowest, which concurs with the observation of RCI slow convergence in [3]. Note that the existing ADMM algorithms for P2P energy systems require either a central coordinator (i.e. not decentralized), or a sequential update of local ADMM variables at each prosumer though all prosumers work in parallel (potentially longer computational time).

In all existing studies on P2P energy systems, two fundamental issues are commonly assumed, one is the successful trading of all peers, and the other is the right selection of cost function parameters by each prosumer. However, the first assumption can be violated in realistic situations because of: (i) distinct expectations between prosumers on the amount of powers and their prices to be traded; (ii) different energy preferences from prosumers. Such violation results in completely different solutions with those when the assumption is satisfied, as will be shown later in this paper. The second assumption is also difficult to be satisfied in reality, because prosumers cannot know exactly the values of their cost function parameters for obtaining their expected power trading amount and price.

This paper aims to fulfill the above research gaps, thereby contributes the following to the P2P energy systems research.

- A decentralized, scalable ADMM approach with parallel updates of prosumers/peers, which also updates local ADMM variables at each prosumer/agent in parallel, for trading in P2P energy market. Thus, it is suitable for distributed and parallel computing platforms, and its computational time is shorter.

- Decentralized learning strategies for tuning parameters of prosumers cost functions to obtain successful trading with expected amount of total traded power.

The rest of this paper is organized as follows. Section 2] introduces the optimal energy management problem in $\mathrm{P} 2 \mathrm{P}$ systems and characterizes its optimal solution in relation with the optimal solution of the pool-based market. Next, a decentralized P2P negotiation mechanism and decentralized learning strategies for prosumers are proposed in Section 3. Case studies are then introduced in Section 4 to illustrate the proposed approaches. Lastly, Section 5 concludes the paper and provides directions for future research.

\section{P2P Electricity Trading Problem}

Consider the P2P energy trading during the time interval $[1, \mathbb{T}]$ for a power system consisting of $n$ prosumers, where each prosumer is regarded as a peer or agent who not only consumes power but also can produce power with some sort of power generation or storage. It is assumed that prosumers behave nonstrategically, i.e. they do not try to learn the other prosumers' behaviors through the trading process.

Denote $P_{i j}(t)$ the power to be traded at time step $t$ between the $i$-th and $j$-th prosumers, where $P_{i j}(t)>$ 0 means prosumer $i$ buys electricity from prosumer $j$, and vice versa, $P_{i j}(t)<0$ means prosumer $i$ sells electricity to prosumer $j$. To simplify the trading of prosumers, it is assumed that at each time step a prosumer only buys or sells power, but not to do both. In other words, at each time step a prosumer holds only one role, an energy buyer or an energy seller.

\subsection{Inter-peer Communication Struc- ture}

Denote $\mathcal{G}(t)$ the inter-prosumer communication graph at time step $t$. Due to the bilateral trading 
between prosumers, $\mathcal{G}(t)$ is undirected. Moreover, $\mathcal{G}(t)$ is a bipartite graph whose node set composes of two disjoint subsets associated to selling and buying prosumers, instead of a fully connected graph (c.f. 323]). Each node only communicates with other nodes in another subset, and do not communicate with nodes inside the same subset.

For each prosumer $i$, denote $\mathcal{N}_{i}(t)$ its neighboring set, i.e. the set of other communicated prosumers for energy trading at time step $t$. Let $E(t)$ denote the incidence matrix of $\mathcal{G}(t)$ associated with an arbitrary edge orientation. Next, let $a_{i j}$ be elements of the adjacency matrix $\mathcal{A}(t)$, i.e. $a_{i j}(t)=1$ if prosumers $i$ and $j$ are connected at time step $t$, and $a_{i j}(t)=0$ otherwise. The degree matrix $\mathcal{D}(t)$ is defined by $\mathcal{D}(t)=\operatorname{diag}\left\{d_{i}(t)\right\}_{i=1, \ldots, n}$, where $d_{i}(t) \triangleq$ $\sum_{j \in \mathcal{N}_{i}(t)} a_{i j}(t)$. Then the Laplacian matrix $\mathcal{L}(t)$ associated to $\mathcal{G}(t)$ is defined by $\mathcal{L}(t)=\mathcal{D}(t)-\mathcal{A}(t)$.

\subsection{Objective Function}

Let $n_{i}(t) \triangleq\left|\mathcal{N}_{i}(t)\right|, P_{i}(t) \in \mathbb{R}^{n_{i}(t)}$ be the vector of all $P_{i j}(t)$ with $j \in \mathcal{N}_{i}(t), P_{i, t r}(t)$ be its total traded power. Then $P_{i, t r}(t)=\mathbf{1}_{n_{i}(t)}^{T} P_{i}(t)$. Next, denote $C_{i}\left(P_{i}(t)\right)$ the total cost of prosumer $i$ for trading in the P2P market, which composes of three components assumed to have the following forms.

$$
\begin{aligned}
& C_{i, 1}\left(P_{i}(t)\right)=a_{i}(t) P_{i, t r}^{2}(t)+\tilde{b}_{i}(t) P_{i, t r}(t) \\
& C_{i, 2}\left(P_{i}(t)\right)=\sum_{j \in \mathcal{N}_{i}} d_{i j} P_{i j}(t) \\
& C_{i, 3}\left(P_{i}(t)\right)=\beta P_{i, t r}(t)
\end{aligned}
$$

The first component (1a) is an utility function whose parameters $a_{i}(t)>0$ and $\tilde{b}_{i}(t)$ are available only for prosumer $i$, which are presented here as timedependent parameters to reflect the time-varying and complex behaviors of prosumers. The second element (1b) is a bilateral trading cost associated with the traded powers with other prosumers, where $d_{i j}$ is the bilateral trade weight (also called trading coefficient in [23]) enforced on the trading between prosumer $i$ and prosumer $j$ for the purposes of product differentiation and consumer involvement 23. For example, renewable and clean power would be preferred to fossil-based power for reducing emissions of pollutants, hence $d_{i j}$ associated with the former is smaller than that corresponding to the latter. The last component (1c) is the implementation cost for the traded powers to be physically executed through the power network, where $\beta>0$ is a fixed rate.

Therefore, summing up (1a)-(1c) gives us the following total cost of each prosumer in the P2P market,

$C_{i}\left(P_{i}(t)\right)=a_{i}(t) P_{i, t r}^{2}(t)+b_{i}(t) P_{i, t r}(t)+\sum_{j \in \mathcal{N}_{i}} d_{i j} P_{i j}(t)$

where $b_{i}(t) \triangleq \tilde{b}_{i}(t)+\beta$. The assumed formula of $C_{i}\left(P_{i}(t)\right)$ above guarantees that it is convex.

\subsection{System Constraints}

The first constraint is on the bilateral trading power, i.e.,

$$
P_{i j}(t)+P_{j i}(t)=0 \forall j \in \mathcal{N}_{i}(t), t=1, \ldots, \mathbb{T}
$$

The next constraint is on the limits of power can be traded,

$$
P_{i, t r}^{\min } \leq P_{i, t r}(t) \leq P_{i, t r}^{\max } \forall t=1, \ldots, \mathbb{T}
$$

Note that power flow constraints are not considered here for the sake of simplifying the analytical analysis, and it is assumed that the cost (1c) is paid to the power network operator for dealing with such flow constraints.

\subsection{Overall Optimization Problem}

The optimal clearing strategy for P2P energy trading is formulated as an optimization problem below.

$$
\begin{aligned}
\min & \sum_{t=1}^{\mathbb{T}} \sum_{i=1}^{n} C_{i}\left(P_{i}(t)\right) \\
\text { s.t. } & P_{i j}(t)+P_{j i}(t)=0 \forall j \in \mathcal{N}_{i}(t) \\
& P_{i, t r}^{\min } \leq P_{i, t r}(t)=\sum_{j \in \mathcal{N}_{i}(t)} P_{i j}(t) \leq P_{i, t r}^{\max } \\
& P_{i j}(t) \leq(\geq) 0 \text { if peer } i \text { is a seller (buyer) } \forall j \in \mathcal{N}_{i}(t)
\end{aligned}
$$


Since the cost functions $C_{i}\left(P_{i}(t)\right)$ are convex and the constraints are linear, the mathematical programming (5) is convex. It can be seen that (5) is decomposable with respect to time index, moreover the role of each prosumer can change between a buyer and a seller from time to time, thus hereafter we will solve (5) at each time step and omit the time index.

In the pool-based market, the optimal energy management problem at each time step $t$ is a social welfare maximization problem, as follows.

$$
\begin{aligned}
& \min \sum_{i=1}^{n} C_{i}\left(P_{i, t r}\right) \\
& \text { s.t. } \sum_{i=1}^{n} P_{i, t r}=0 \\
& P_{i, t r}^{\min } \leq P_{i, t r} \leq P_{i, t r}^{\max } \\
& P_{i, t r} \leq(\geq) 0 \text { if peer } i \text { is a seller (buyer) } \forall j \in \mathcal{N}_{i}(t)
\end{aligned}
$$

Solutions of the P2P market problem (5) and the pool-based market problem (6) will be compared to show the differences between these two markets.

Remark 1 Time binding constraints, for example

$$
P_{i, t r}^{r, \min }(t) \leq P_{i, t r}(t)-P_{i, t r}(t-1) \leq P_{i, t r}^{r, \max }(t)
$$

where $P_{i, t r}^{r, \min }(t)$ and $P_{i, t r}^{r, \max }(t)$ are obtained from the predicted generation and consumption of prosumer $i$ at time step $t$, can be included in the P2P optimization problem (5). Then solving (5) for all time steps at once is suitable for day-ahead or longer scheduling problems. However, this research focuses on the P2P energy trading one-time-ahead, and therefore solve (5) consecutively at each time step. As such, at time step $t, P_{i, t r}(t-1)$ is known, hence (17) can be rewritten as

$P_{i, t r}^{r, \min }(t)+P_{i, t r}(t-1) \leq P_{i, t r}(t) \leq P_{i, t r}^{r, \max }(t)+P_{i, t r}(t-1)$ which is similar to (5c), and therefore can be combined into a unique constraint. Note that solving P2P optimization problem (5) in real-time would be impractical in real-world systems due to the large scale of the system and possible latencies on interpeer communications. Thus, the interval between two consecutive time steps is usually an hour or a half hour in real-world systems.

\subsection{Characterization of Optimal Solu- tion}

Define the following Lagrangian associated to (5),

$$
L\left(P_{i j}, \lambda_{i j}\right)=\sum_{i=1}^{n} C_{i}\left(P_{i}\right)-\sum_{i=1}^{n} \sum_{j \in \mathcal{N}_{i}} \lambda_{i j}\left(P_{i j}+P_{j i}\right)
$$

where $\lambda_{i j}$ are the Lagrange multipliers associated to the power trading equations (5b), which are regarded as the market clearing prices for energy transactions between pairs of prosumers. To essentially compare the solution of the P2P market (5) with the poolbased market (6), only the equality constraints are considered in the following, since the inequality constraints in those problems are the same. The KKT conditions read as,

$$
\begin{aligned}
\left.\frac{\partial C_{i}}{\partial P_{i j}}\right|_{P_{i j}^{*}} & =\lambda_{i j}^{*} \forall j \in \mathcal{N}_{i} \\
P_{i j}^{*}+P_{j i}^{*} & =0 \forall j \in \mathcal{N}_{i}
\end{aligned}
$$

Condition (9a) leads to

$$
2 a_{i} P_{i, t r}^{*}+b_{i}+d_{i j}=\lambda_{i j}^{*} \forall j \in \mathcal{N}_{i}
$$

which shows that the trade weights $d_{i j}$ are parts of the prices $\lambda_{i j}$. Denote

$P_{t r}^{*} \triangleq\left[2 a_{1} P_{1, t r}^{*}, \ldots, 2 a_{n} P_{n, t r}^{*}\right]^{T}, \alpha \triangleq\left[\frac{1}{2 a_{1}}, \ldots, \frac{1}{2 a_{n}}\right]^{T}$

Theorem 1 The following statements hold.

(i) If no trade weights are imposed, i.e. $d_{i j}=$ $0 \forall i, j=1, \ldots, n$, and the communication graph between successfully traded peers is connected, then all transaction prices are the same and equal to

$$
\lambda^{*}=\frac{\sum b_{j} /\left(2 a_{j}\right)}{\sum 1 /\left(2 a_{j}\right)}
$$

The optimal total trading power for each peer is

$$
P_{i, t r}^{*}=\frac{\sum b_{j} /\left(2 a_{j}\right)}{2 a_{i} \sum 1 /\left(2 a_{j}\right)}-\frac{b_{i}}{2 a_{i}}
$$

The sub-indexes in (11) and (12) are taken for successfully traded peers. Moreover, these optimal price and total traded powers are the same 
with the optimal solutions of the social welfare maximization problem (6).

(ii) If $d_{i j}=0 \forall i, j=1, \ldots, n$, and the communication graph between successfully traded peers is unconnected, then the considering P2P market is clustered into smaller P2P markets, each of them has a different energy price. The optimal price and traded powers for each smaller P2P market are calculated similarly to (11) and (12).

(iii) If $d_{i j} \neq 0$, then energy prices for successful transactions are different from each other. The optimal total traded powers are computed from

$$
[E, \alpha]^{T} P_{t r}^{*}=\left[\operatorname{vec}\left(b_{j}+d_{j i}-b_{i}-d_{i j}\right)^{T}, 0\right]^{T}
$$

Proof. See Appendix.

It is worth emphasizing that the above classical Lagrangian method does not give us a way to compute the individual traded power $P_{i j}$ in each transaction, instead it only provides the total traded power $P_{i, t r}$. Further, as seen in Theorem 1 the optimal energy prices and trading powers are dependent only on successfully traded prosumers, which, in realistic situations, could be a subset of all participated prosumers. Thus, in the next section, a decentralized approach is proposed to analytically derive the power amount $P_{i j}$ and energy price in each successful transaction in $\mathrm{P} 2 \mathrm{P}$ energy market. Then decentralized learning methods are proposed to tune the prosumers cost function parameters such that all of them can successfully trade with desired power amounts.

\section{P2P Energy Negotiation Mechanism and Prosumer Learning Strategy}

\subsection{Decentralized P2P Negotiation Mechanism}

In the following, a decentralized ADMM approach is proposed to solve the mathematical programming (5) in parallel. The advantage of this approach is that it allows each prosumer (peer) to solve its own local optimization problem while negotiating with other prosumers to eventually reach the solution of the global optimization problem (5). Thus, the communication and computation burden at a central entity is avoided, and the privacy of each prosumer can be better guaranteed.

It should be noted that the classical two-block ADMM method [5] is centralized (could be implemented in a distributed manner), and the updates of variables are in order. On the other hand, the existing multi-block ADMM approach 7 allows variables to be updated in parallel, but requires fully connected inter-agent communication which is not fitted into the bipartite structure of the considering $\mathrm{P} 2 \mathrm{P}$ energy system. Therefore, in this research, a novel decentralized ADMM approach is proposed for the $\mathrm{P} 2 \mathrm{P}$ energy market that solves (18) in parallel at all prosumers, and local variables at each prosumer are also updated in parallel.

Denote $m \triangleq \sum_{i=1}^{n} n_{i}, P \in \mathbb{R}^{m}$ the vector of all $P_{i}, i=1, \ldots, n$, and the sets of equality constraint (5b) and inequality constraint (5c) as in (14) and (15), respectively.

$$
\begin{gathered}
\Omega_{e q} \triangleq\left\{P \in \mathbb{R}^{m}: P_{i j}(t)+P_{j i}(t)=0 \forall j \in \mathcal{N}_{i}\right\} \\
\Omega_{\text {ineq }} \triangleq\left\{P \in \mathbb{R}^{m}: P_{i, t r}^{\min } \leq \mathbf{1}_{n_{i}}^{T} P_{i} \leq P_{i, t r}^{\max }\right\}
\end{gathered}
$$

For those sets, the following indicator functions are defined.

$$
\begin{gathered}
I_{e q}(P) \triangleq\left\{\begin{aligned}
0: & P \in \Omega_{e q} \\
+\infty: & P \notin \Omega_{e q}
\end{aligned}\right. \\
I_{\text {ineq }}(P) \triangleq\left\{\begin{aligned}
0: & P \in \Omega_{\text {ineq }} \\
+\infty: & P \notin \Omega_{\text {ineq }}
\end{aligned}\right.
\end{gathered}
$$

Now, by utilizing a new variable $X \in \mathbb{R}^{m}$, the optimization problem (5) is rewritten such that equality and inequality constraints are separated into different sets corresponding to different variables $P$ and $X$, as follows.

$$
\begin{aligned}
\min & \sum_{i=1}^{n} C_{i}\left(P_{i}\right)+I_{e q}(P)+I_{\text {ineq }}(X) \\
\text { s.t. } & P-X=0 \\
& P \in \Omega_{e q}, X \in \Omega_{\text {ineq }}
\end{aligned}
$$


Obviously, (18) is in the standard form of the ADMM method [5]. Define the following augmented Lagrangian,

$$
\begin{aligned}
L_{\rho}(P, X, u)= & \sum_{i=1}^{n} C_{i}\left(P_{i}\right)+I_{e q}(P)+I_{\text {ineq }}(X) \\
& +\frac{\rho}{2}\|P-X+u\|_{2}^{2}
\end{aligned}
$$

where $\rho>0$ is a scalar penalty parameter and $u \in$ $\mathbb{R}^{m}$ is called the scaled Lagrange (dual) multiplier [5]. Next, the variables $P, X, u$ are computed in parallel at each algorithm iteration $k+1$ by solving the following sub-problems,

$$
X^{k+1} \triangleq \underset{X \in \Omega_{\text {ineq }}}{\operatorname{argmin}} L_{\rho}\left(P^{k}, X, u^{k}\right)+\frac{1}{2}\left(X-X^{k}\right)^{T} \Psi(X-X
$$$$
P^{k+1} \triangleq \underset{P \in \Omega_{e q}}{\operatorname{argmin}} L_{\rho}\left(P, X^{k}, u^{k}\right)+\frac{1}{2}\left(P-P^{k}\right)^{T} \Phi\left(P-P^{k}\right)
$$

$$
u^{k+1} \triangleq u^{k}+\kappa \rho\left(P^{k+1}-X^{k+1}\right)
$$

in which $\Phi, \Psi, \kappa>0$ satisfy

$$
\Phi \succ \rho\left(\frac{1}{\mu_{1}}-1\right) I, \Psi \succ \rho\left(\frac{1}{\mu_{2}}-1\right) I, \mu_{1}+\mu_{2}<2-\kappa
$$

for some $\mu_{1}>0, \mu_{2}>0$. Condition (20) was proved to be sufficient for the convergence of the above variables update [7]. Note that the selection of $\Phi, \Psi, \kappa$ to fulfill (20) is not unique. One simple way is to let $\Phi=\phi I, \Psi=\psi I$ such that

$$
\phi>\rho\left(\frac{1}{\mu_{1}}-1\right), \psi>\rho\left(\frac{1}{\mu_{2}}-1\right), \mu_{1}+\mu_{2}<2-\kappa
$$

There are also multiple choices of $\mu_{1}, \mu_{2}$ and $\kappa$ to satisfy (21). For instance, let $\mu_{1}=\mu_{2}=0.5$, then (21) becomes $\phi>\rho, \psi>\rho, \kappa<1$, which will be used in the case studies later.

Stopping criteria for the iterative process above are [5]

$$
\left\|r^{k}\right\|_{2} \leq \epsilon^{\mathrm{pri}},\left\|s^{k}\right\|_{2} \leq \epsilon^{\mathrm{dual}}
$$

where $r^{k} \triangleq P^{k}-X^{k}$ and $s^{k} \triangleq-\rho\left(X^{k}-X^{k-1}\right)$ are primal and dual residuals at iteration $k$; $\epsilon^{\text {pri }}>0$ and $\epsilon^{\text {dual }}>0$ are primal and dual feasibility tolerances chosen by [5]

$$
\begin{aligned}
\epsilon^{\mathrm{pri}} & =\sqrt{n+m} \epsilon^{\mathrm{abs}}+\epsilon^{\mathrm{rel}} \max \left\{\left\|P^{k}\right\|_{2},\left\|-X^{k}\right\|_{2}\right\}, \\
\epsilon^{\mathrm{dual}} & =\sqrt{n+m} \epsilon^{\mathrm{abs}}+\epsilon^{\mathrm{rel}}\left\|\rho u^{k}\right\|_{2},
\end{aligned}
$$

Here, $\epsilon^{\mathrm{abs}}>0$ and $\epsilon^{\mathrm{rel}}>0$ are absolute and relative tolerances, set in this research to be $10^{-4}$ and $10^{-3}$, respectively. The verification of (22) in a decentralized manner were presented in [21, Theorem 2], hence is omitted here for brevity.

The updates of variables $P$ and $X$ in (19) are dependent only on their values at the previous iteration, hence can be made without any order, unlike other existing ADMM algorithms for P2P energy systems in the literature, e.g. 3, 4, 15, 18, 29, where variables Xde)pend on the others at the current iteration and must be updated one after another.

\subsubsection{The Update for Variable $X$}

The update for $X^{k+1}$ in (19) is derived by solving the following optimization problem.

$$
\begin{aligned}
& \min \frac{\rho}{2}\left\|P^{k}-X+u^{k}\right\|_{2}^{2}+\frac{\psi}{2}\left\|X-X^{k}\right\|_{2}^{2} \\
& \text { s.t. } P_{i, t r}^{\min } \leq \mathbf{1}_{n_{i}}^{T} X_{i} \leq P_{i, t r}^{\max } \forall i=1, \ldots, n
\end{aligned}
$$

Note that there is one more constraint on the positiveness or negativeness of each $X_{i}, i=1, \ldots, n$, depending on whether prosumer $i$ at the next time slot will perform as a buyer or seller. In any case, (24) is a quadratic convex problem and is decomposable to each prosumer/peer, i.e. it is fully decentralized, hence it can be easily solved by any off-the-self software embedded in each prosumer/peer, e.g. CVX [1].

\subsubsection{The Update for Variable $P$}

To obtain the update for $P^{k+1}$ in (19), we need to solve the following mathematical programming.

$$
\begin{aligned}
& \min \sum_{i=1}^{n} C_{i}\left(P_{i}\right)+\frac{\rho}{2}\left\|P-X^{k}+u^{k}\right\|_{2}^{2}+\frac{\phi}{2}\left\|P-P^{k}\right\|_{2}^{2} \\
& \text { s.t. } P_{i j}+P_{j i}=0 \forall i=1, \ldots, n ; j \in \mathcal{N}_{i}
\end{aligned}
$$


Denote $\lambda_{i j}>0$ the Lagrange multiplier associated with the constraint (25b), and $\lambda_{i} \in \mathbb{R}^{n_{i}}$ the vector of all $\lambda_{i j}$ with $j \in \mathcal{N}_{i}$. Since (25) is a convex optimization problem with quadratic cost function and linear equality, the strong duality holds and KKT conditions apply. Therefore, we obtain from (25) that

$$
\begin{aligned}
\lambda_{i j}^{k+1}= & \frac{\partial}{\partial P_{i j}}\left(\sum_{i=1}^{n} C_{i}\left(P_{i}\right)+\frac{\rho}{2}\left\|P-X^{k}+u^{k}\right\|_{2}^{2}\right. \\
& \left.+\frac{\phi}{2}\left\|P-P^{k}\right\|_{2}^{2}\right)\left.\right|_{P_{i j}=P_{i j}^{k+1}} \\
= & 2 a_{i} P_{i, t r}^{k+1}+(\rho+\phi) P_{i j}^{k+1}+v_{i j}^{k}
\end{aligned}
$$

where $v_{i j}^{k} \triangleq b_{i}+d_{i j}+\rho\left(-X_{i j}^{k}+u_{i j}^{k}\right)-\phi P_{i j}^{k}$. Next, due to the bilateral trading constraint (25b), the Lagrange multipliers and the traded powers must satisfy the following constraints.

$$
\lambda_{i j}^{k+1}=\lambda_{j i}^{k+1}, P_{i j}^{k+1}=-P_{j i}^{k+1} \forall j \in \mathcal{N}_{i}
$$

Here, $\lambda_{i j}^{k+1}$ is considered to be the trading price between the $i$-th and $j$-th prosumers. Denote

$$
\begin{aligned}
\hat{v}_{i}^{k+1} & \triangleq \sum_{j \in \mathcal{N}_{i}} v_{j i}^{k+1}, \tilde{v}_{i}^{k+1} \triangleq \sum_{j \in \mathcal{N}_{i}} v_{i j}^{k+1} \\
\hat{v}^{k+1} & \triangleq\left[\hat{v}_{1}^{k+1}, \cdots, \hat{v}_{n}^{k+1}\right]^{T}, \tilde{v}^{k+1} \triangleq\left[\tilde{v}_{1}^{k+1}, \cdots, \tilde{v}_{n}^{k+1}\right]^{T} \\
\Gamma & \triangleq(\rho+\phi) \operatorname{diag}\left\{\frac{1}{a_{i}}\right\}_{i=1, \ldots, n}
\end{aligned}
$$

Theorem 2 The iterative update for individual power and energy transaction price are as follows.

$$
\begin{aligned}
P_{i j}^{k+1} & =\frac{v_{j i}^{k+1}+2 a_{j} P_{j, t r}^{k+1}-v_{i j}^{k+1}-2 a_{i} P_{i, t r}^{k+1}}{2(\rho+\phi)} \\
\lambda_{i j}^{k+1} & =\frac{v_{j i}^{k+1}+2 a_{j} P_{j, t r}^{k+1}+v_{i j}^{k+1}+2 a_{i} P_{i, t r}^{k+1}}{2}
\end{aligned}
$$

where $P_{i, t r}^{k+1}$ is computed from

$$
(\mathcal{L}+\Gamma) \operatorname{vec}\left(2 a_{i} P_{i, t r}^{k+1}\right)=\hat{v}^{k+1}-\tilde{v}^{k+1}
$$

As $k \rightarrow \infty$ and $\rho \rightarrow 0$, the optimal total traded power and optimal energy price converge to that stated in Theorem 1 .
Proof. See Appendix.

Remark 2 Equation (29) can be solved in a decentralized manner as a decentralized least-square problem using several methods, e.g. [11, 14]. Details are ignored here for brevity.

\subsection{Prosumer Cost Function Parame- ters Tuning by Learning}

\subsubsection{Learn For Successful Trading}

As discussed before, it is possible that not all participated prosumers successfully trade. Therefore, this section proposes a simple but effective learning strategy for prosumers (peers) to adjust their cost function parameters to obtain successful energy transaction, when their previous attempt was failed.

One possible reason for negotiation failure is the dissatisfaction of energy price and the constraint $P_{i, t r}^{*}>0\left(P_{i, t r}^{*}<0\right)$ for buying (selling) prosumer $i$, which lead to $P_{i, t r}^{*}=0$. Hence, the proposed strategy is based on the analytical formula (12) of the optimally traded powers to change prosumers parameters. Recalling from (12) that

$$
P_{i, t r}^{*}=\frac{\sum b_{j} /\left(2 a_{j}\right)}{2 a_{i} \sum 1 /\left(2 a_{j}\right)}-\frac{b_{i}}{2 a_{i}}=\frac{\sum\left(b_{j}-b_{i}\right) /\left(2 a_{j}\right)}{2 a_{i} \sum 1 /\left(2 a_{j}\right)}
$$

Consequently, the unsuccessfully traded prosumers keep their parameters $a_{i}$ unchanged, while decreasing (increasing) their parameters $b_{i}$ if they are buying (selling) prosumers. As such, $P_{i, t r}^{*}$ will be increased (decreased) for buying (selling) prosumers. This process is repeated, and the increase or decrease of $b_{i}$ at each repetition can be selected to be a constant, for simplicity. Then unsuccessfully bought (sold) prosumers will eventually get $P_{i, t r}^{*}>0\left(P_{i, t r}^{*}<0\right)$, i.e. successful transactions.

This learning procedure can also be physically explained as follows. The quantity $2 a_{i} P_{i, t r}^{*}+b_{i}$, which is the partial derivative of prosumer $i$ cost function $C_{i}(\cdot)$ with respect to $P_{i, t r}$ at its optimal value, is often regarded as an optimal marginal cost. Therefore, if prosumer $i$ is a buyer who decreases $b_{i}$ while keeping $a_{i}$ unchanged, then his marginal cost decreases, 
i.e. he can get more profit. Similarly, if prosumer $j$ is a seller who can increase $b_{j}$ and hold $a_{j}$ unchanged, then his marginal cost increases, i.e. he is willing to lower his profit. As a result, following the proposed learning method, prosumers will gradually match the expected profits of the others, and achieve successful energy transactions.

\subsubsection{Learn For Increasing Traded Power Amount}

Employing (30), there are two possible ways to boost the amounts of traded powers: (i) increase $b_{i}$; (ii) decrease $a_{i}$, for instance by a magnitude of $\gamma$, with $\gamma>1$. While both the former and the latter method can boost the traded amounts of powers by $\gamma$ times, the former obviously rises the trading price due to the increase of $v_{i j}$ and $P_{i j}$ in (28b). On the other hand, the latter does not necessarily increase the trading price, because $b_{i}$ and $a_{i} P_{i j}$ remain the same in (28b). Thus, the latter method is selected as the learning method for increasing the amounts of traded power in $\mathrm{P} 2 \mathrm{P}$ energy market.

Finally, the proposed decentralized P2P ADMM algorithm and decentralized cost function parameters learning methods are summarized in Figure 1 .

\section{Case Studies}

\subsection{Synthetic System}

This section aims at illustrating the effectiveness of the proposed decentralized optimization algorithm and decentralized learning strategies for P2P energy trading on a synthetic example. A system of 6 prosumers is considered with the parameters given in Table 1. The parameters $a_{i}, b_{i}$ of prosumers are inspired by that of generators reported in the literature (e.g. that in 21] and references therein). P2P energy trading is assumed to occur every hour.

Six scenarios will be considered. Scenarios 1 examines the solutions of the pool-based market and compares them with the P2P market solutions. Then scenarios 2 and 3 with different inter-peer communication structures will be investigated (see first two

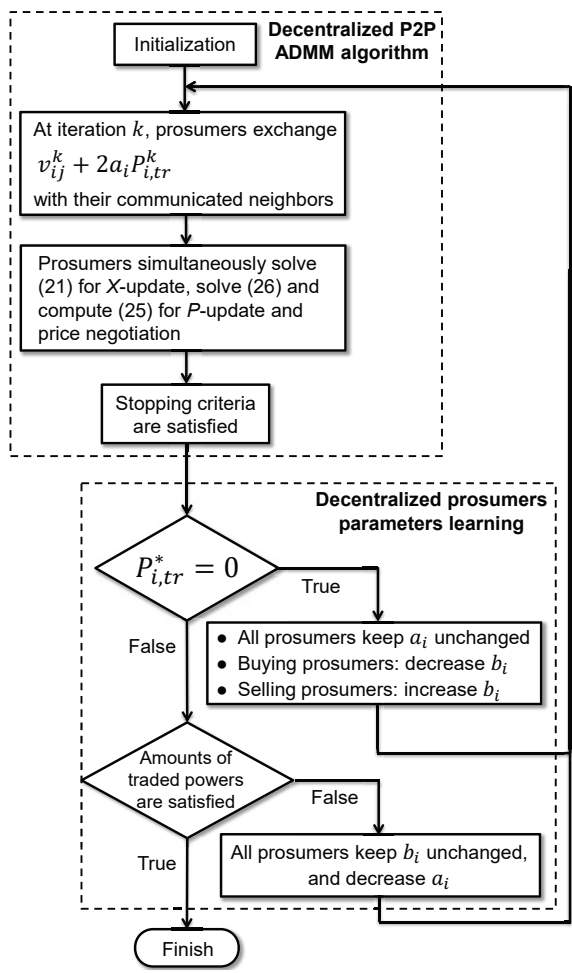

Figure 1: The proposed distributed P2P ADMM optimization and decentralized prosumers cost function paramters learning approach at a time step.

Table 1: Parameters for synthetic system.

\begin{tabular}{|c|c|c|c|c|c|c|}
\hline Prosumer & 1 & 2 & 3 & 4 & 5 & 6 \\
\hline$a_{i}$ & 0.0031 & 0.0074 & 0.0066 & 0.0063 & 0.0069 & 0.0095 \\
\hline$b_{i}$ & 8.71 & 3.53 & 7.58 & 2.24 & 8.53 & 3.46 \\
\hline$P_{i, t r}^{\min }[\mathrm{kW}]$ & -105 & -115 & -125 & 0.01 & 0.01 & 0.01 \\
\hline$P_{i, t r}^{\max }[\mathrm{kW}]$ & -0.01 & -0.01 & -0.01 & 100 & 110 & 95 \\
\hline
\end{tabular}



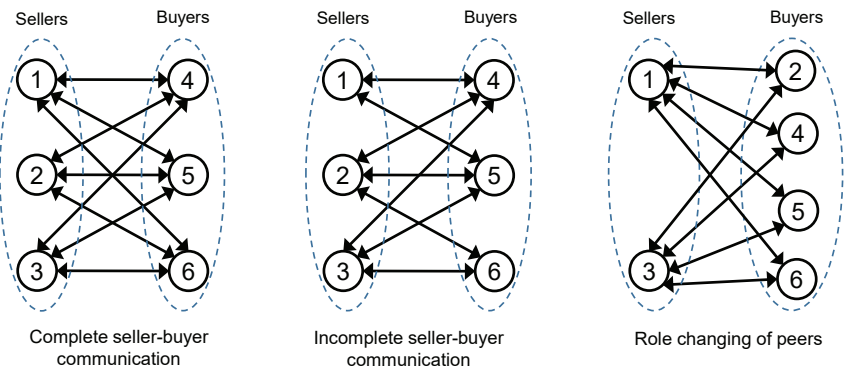

Figure 2: Time-varying inter-peer communication structure.

graphs in Figure 21). Next, in the 4th scenario, results of $\mathrm{P} 2 \mathrm{P}$ market when a prosumer changes its role will be presented (see the last graph in Figure 2). These four scenarios do not consider the bilateral trade weights $d_{i j}$, hence scenario 5 will investigate the effects of such weights. Lastly, scenario 6 demonstrates the proposed decentralized learning strategies in Section 3.2 such that all prosumers successfully trade. In all illustrating figures, dash lines represent energy transaction prices, whereas solid lines show traded powers. All simulations are conducted in MATLAB installed on a computer equipped with Intel Core i7-6700K CPU 4GHz and 64GB RAM. Optimization problem (24) for variable $X$ update is solved by the software CVX [1]. All variables are initialized at zero.

\subsubsection{Scenario 1 (Pool-based market)}

Decentralized ADMM approaches for solving the problem (6) without P2P energy trading was proposed in our previous works [20,21. The results presented in Figure 3 are obtained using the algorithm in [20] with $\rho=0.02, \phi=0.021, \psi=0.021, \kappa=0.99$.

\subsubsection{Scenario 2 (P2P market with buyer- seller complete communication)}

In this scenario, each buying prosumer is communicated with each selling prosumer, as displayed in the first graph in Figure 2, Parameters used in the proposed Decentralized ADMM algorithm are the same

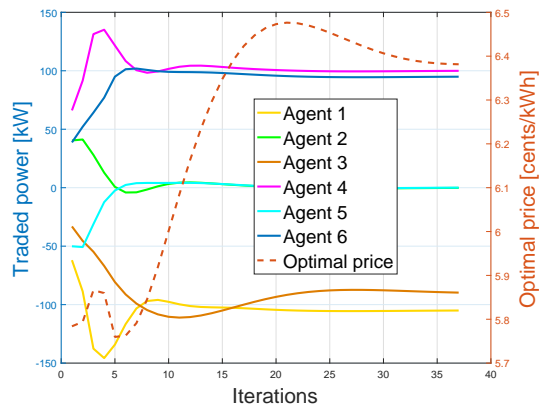

Figure 3: Power trading in Scenario 1.

with scenario 1. Simulation results depicted in Figure 4 show that peer 2 and peer 5 do not trade, but energy prices of all successful transactions are identical, because the communication graph between successfully traded peers is connected. Additionally, the total traded power of each peer and energy price are the same with that obtained in scenario 1 . This confirms the first statement of Theorem 1

\subsubsection{Scenario 3 (P2P market with buyer- seller incomplete communication)}

Suppose that the communication link between the 1st and 6th prosumers is removed, as seen in the second graph in Figure 2 ADMM parameters are the same with scenario 2. Simulation results for this scenario are then shown in Figure 5 Similar to scenario 2 , peer 2 and peer 5 also do not successfully trade. On the other hand, peer 1 only sells power to peer 4 , whilst peer 3 only sells power to peer 6 , hence the communication graph between successfully traded peers is unconnected. This results in a clustered P2P market consisting of two small P2P markets with different energy prices. This result illustrates the 2nd statement of Theorem 1

\subsubsection{Scenario 4 (P2P market with role changing of peers)}

This section demonstrates the P2P market flexibility and time-varying behaviors of prosumers, where a seller, particularly peer 2 , now becomes a buyer. Ac- 

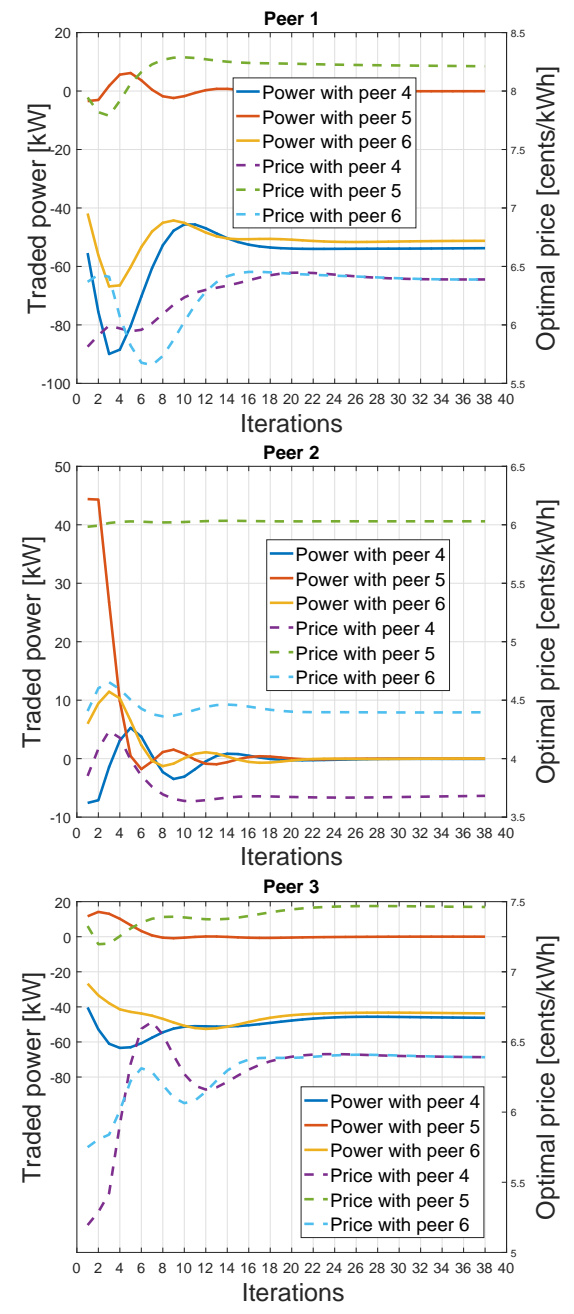

Figure 4: P2P power trading in Scenario 2.
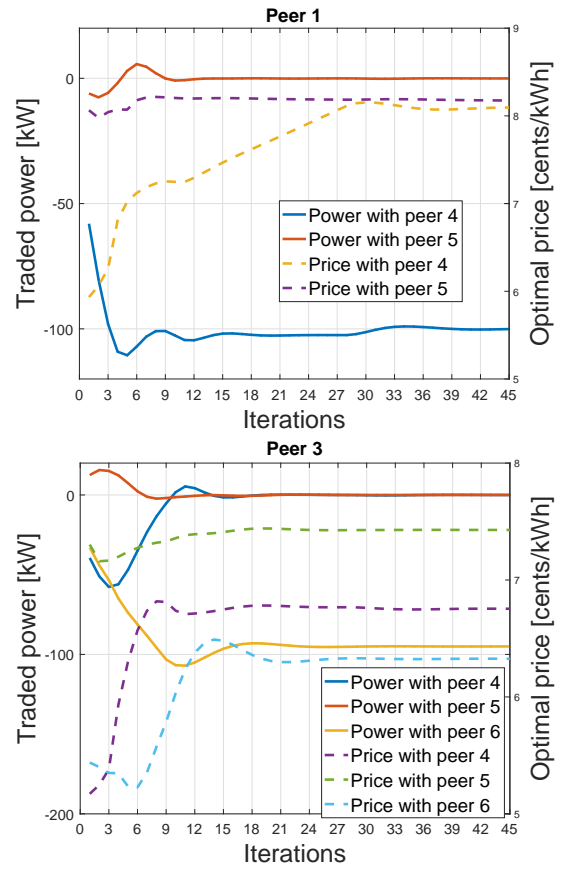

Figure 5: P2P power trading in Scenario 3.

cordingly, lower and upper bounds for traded powers of peer 2 are reversed with opposite signs. ADMM parameters are the same with scenario 2 .

The simulation results are then displayed in Figure 6. Peer 5 again does not trade, like in all previous cases, but peer 2 now buys power from both peer 1 and peer 3 . Peer 4 and peer 6 also buy power from both peer 1 and peer 3 . It is noted that the traded price and powers in this scenario are distinct from that in scenarios 2 and 3 because of peer 2 role changing, which illustrate the time-varying behaviors of prosumers in $\mathrm{P} 2 \mathrm{P}$ energy market.

\subsubsection{Scenario 5 (P2P market with effects of bilateral trade weights)}

The communication structure between buying and selling prosumers in this case is the same with that in scenario 2 , and single-criterion bilateral trade weights are enforced for each transaction based on the energy sources, i.e. renewable or fossil-based. As such, we 

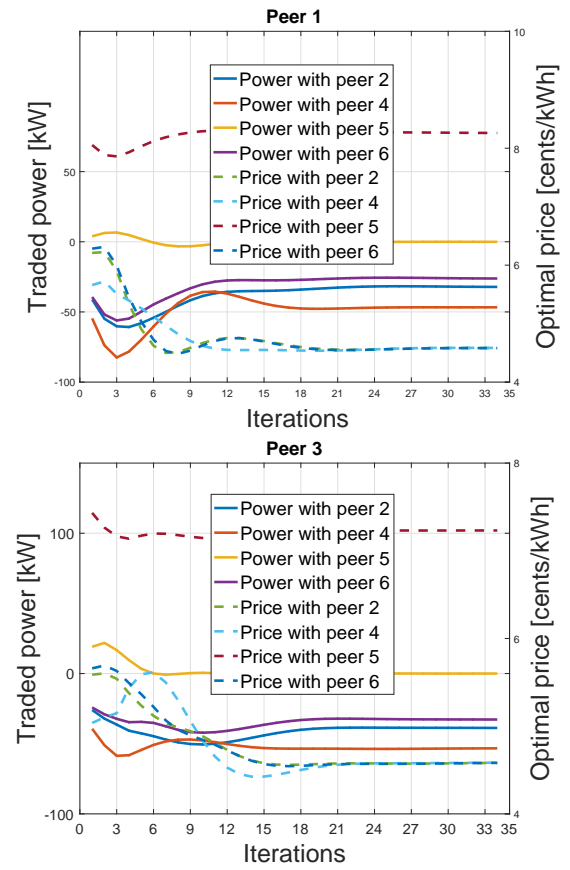

Figure 6: P2P power trading in Scenario 4.

assume that peer 1 is fossil-based producer, whilst peer 2 and peer 3 are renewable producers, and $d_{41}=0.51, d_{51}=0.51, d_{61}=0.72, d_{42}=d_{43}=0.1$, $d_{52}=d_{53}=0.12, d_{62}=d_{63}=0.04$. These parameters show strong preference of buyers to renewable over fossil-based power, and the strongest supporter is peer 6 .

The simulation results in this scenario are then shown in Figure 7 with $\rho=0.009, \phi=0.0091, \psi=$ 0.0091 which give heuristically fastest convergence among many different values of $\rho, \phi, \psi$. It is observed that peers 2 and 5 are unsuccessfully traded, like in scenarios 2-4, while results for other peers are substantially changed because of the trade weights $d_{i j}$, as anticipated in the 3rd statement of Theorem 1 .

First, the energy transaction prices are no longer the same as in Scenario 2, even the buyer-seller communication structure is complete. Second, the trading of each buyer-seller pair are completely different from that in previous cases. Particularly, the power that peer 6 trades with peer 1 becomes very small, 5.1
$\mathrm{kW}$, in comparison with $51.2 \mathrm{~kW}$ in Scenario 2. Peer 6 then turns to peer 3 to buy $90.1 \mathrm{~kW}$ from it. This is fully explainable due to the strong opposition of peer 6 to fossil-based generation (peer 1) while showing high interest in renewable supply (peer 3). Hence, in realistic $\mathrm{P} 2 \mathrm{P}$ energy markets, trade weights can be employed by prosumers to help attain strategic objectives such as emissions reduction, loss decrease, etc., for example by putting small values for renewable and big values for fossil-based sources.
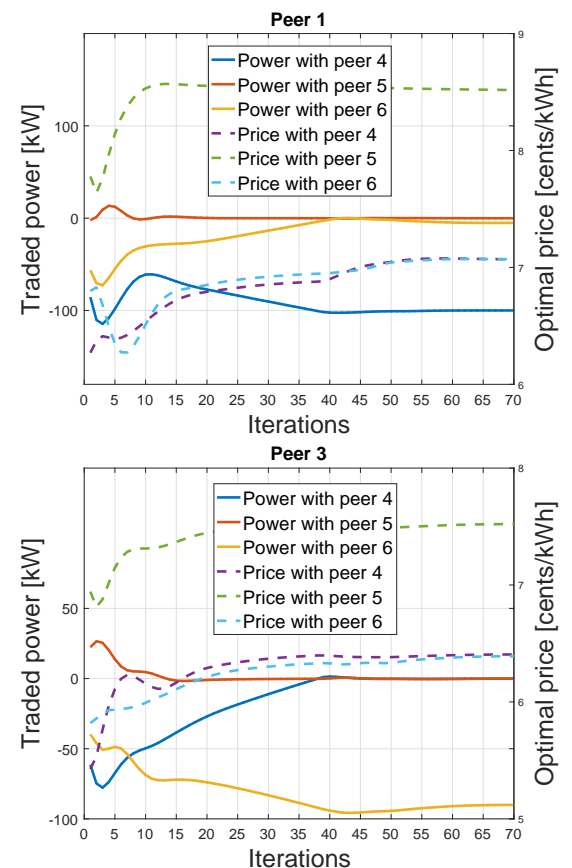

Figure 7: P2P power trading in Scenario 5.

Table 2: Convergence time for synthetic system.

\begin{tabular}{|c|c|c|c|c|c|c|}
\hline Scenario & 1 & 2 & 3 & 4 & 5 & 6 \\
\hline Time & $0.008 \mathrm{~s}$ & $11 \mathrm{~s}$ & $10.6 \mathrm{~s}$ & $7.9 \mathrm{~s}$ & $17.9 \mathrm{~s}$ & $10.3 \mathrm{~s}$ \\
\hline
\end{tabular}


Table 3: Total traded power (in black) and costs (in red) of prosumers in different scenarios.

\begin{tabular}{|c|c|c|c|c|c|c|}
\hline Pros. & Scen.1 & Scen.2 & Scen.3 & Scen.4 & Scen.5 & Scen.6 \\
\hline \multirow{2}{*}{1} & -105 & -105 & -100 & -105 & -105 & -105 \\
\cline { 2 - 7 } & -669.9 & -669.9 & -810 & -480.9 & -742.35 & -649.95 \\
\hline \multirow{2}{*}{2} & 0 & 0 & 0 & 70.93 & 0 & -96.13 \\
\cline { 2 - 7 } & 0 & 0 & 0 & -480.9 & 0 & -595.05 \\
\hline \multirow{2}{*}{3} & -90 & -90 & -95 & -124.83 & -90 & -103.87 \\
\cline { 2 - 7 } & -574.2 & -574.2 & -600.97 & -571.72 & -636.3 & -642.96 \\
\hline \multirow{2}{*}{4} & 100 & 100 & 100 & 100 & 100 & 100 \\
\cline { 2 - 7 } & 638 & 638 & 810 & 458 & 707 & 619 \\
\hline \multirow{2}{*}{5} & 0 & 0 & 0 & 0 & 0 & 110 \\
\cline { 2 - 7 } & 0 & 0 & 0 & 0 & 0 & 680.9 \\
\hline \multirow{2}{*}{6} & 95 & 95 & 95 & 58.9 & 95 & 95 \\
\cline { 2 - 7 } & 606.1 & 606.1 & 600.97 & 269.76 & 671.65 & 588.05 \\
\hline
\end{tabular}

\subsubsection{Scenario 6 (Decentralized learning for leading to more time for convergence. successful trading)}

This section illustrates the decentralized learning strategy for obtaining successful trading proposed in Section 3.2 The inter-peer communication structure is the same with that in scenario 2 . Since peer 2 and peer 5 were failed to trade with other peers in all previously introduced scenarios, the proposed learning strategy is only applied to them. Accordingly, $a_{2}$ and $a_{5}$ are kept unchanged, whereas $b_{2}$ is increased and $b_{5}$ is decreased.

The simulation results exhibited in Figure 8 are obtained when $b_{2}=7.53$ and $b_{5}=4.53$. It can be seen that all peers now successfully trade, which demonstrates the efficiency of the proposed learning strategy.

Next, convergence time (without inter-peer communication time) of the proposed decentralized ADMM algorithm for $\mathrm{P} 2 \mathrm{P}$ trading in all scenarios is shown in Table 2. It can be observed that computational time of scenario 5 with trade weights is longest, while that of scenario 1 with pool-based market is fastest, and that of the others are quite similar. This is logical because in pool-based market, no convex optimization problem is needed to solve in the $X$-update step, unlike that in the $\mathrm{P} 2 \mathrm{P}$ market, and trade weights make bilateral trading asymmetric

Finally, total traded powers and costs for prosumers across all scenarios are provided in Table 3 . Those for scenarios 1 and 2 are the same, as seen before. Total traded costs in scenarios 3 and 4 are significantly different from other cases, as the system is clustered (scenario 3), or a prosumer switches between selling and buying roles (scenario 4). Total traded costs in scenario 6 reveal that prosumers 4 and 6 need to pay less, whereas prosumer 1 suffers a bit loss, and prosumer 3 gains considerably more, compared to scenarios 1 and 2. Hence, the decentralized learning for successful trading of prosumers 2 and 5 not only helps themselves but also is beneficial for many other prosumers, though not all. On the other hand, energy price is increased in scenario 5 though trade weights help change the traded amounts of powers, leading to higher payments for prosumers 4 and 6 , and simultaneously bring more profit to prosumer 1 and 3. Thus, how to choose the best trade weights to enforce on bilateral trading between prosumers needs further investigation. 

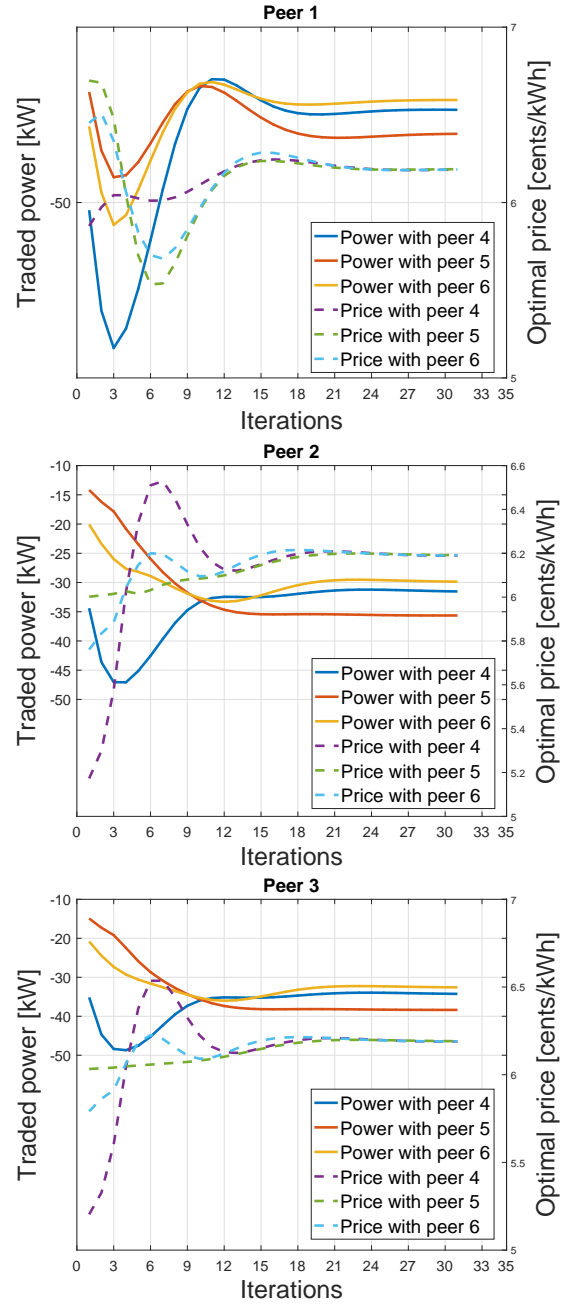

Figure 8: P2P power trading in Scenario 6.

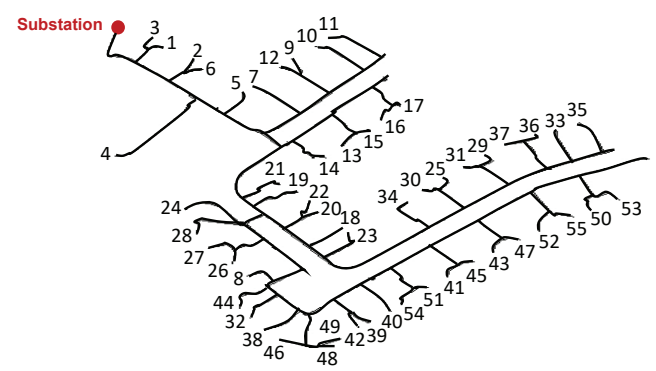

Figure 9: Diagram of the IEEE European Low Voltage Test Feeder.

\subsection{Modified IEEE European Low Voltage Test Feeder}

In this section, validation of the proposed approach is carried out for the IEEE European Low Voltage Test Feeder having 55 nodes 10 shown in Figure 9. It is assumed that 25 nodes have $5.5 \mathrm{~kW}$ rooftop solar systems, while the remaining nodes have $3 \mathrm{kWh}$ battery systems. P2P energy trading is allowed in this system each one-hour interval. One-hour load profiles of 55 nodes are taken from [10], and average daily global solar irradiance data in July in Spain are obtained from [8]. Then power output of $5.5 \mathrm{~kW}$ solar systems are computed using the formula in 22] (see Eq. (6) in 22]), where temperature effect (see Eq. (7) in 22) is ignored because it is very small. Load demands of all 55 nodes and solar power output are depicted in Figure 10.

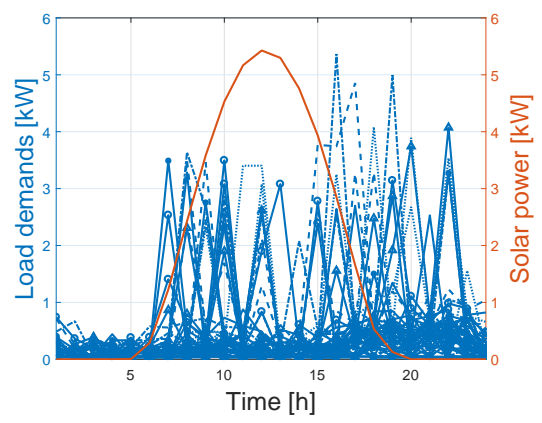

Figure 10: Load demands and assumed solar generation in the IEEE European Low Voltage Test Feeder.

As seen in Figure 10, at noon solar generation is maximum, while 25 solar-equipped nodes have maximum demands of $3.5 \mathrm{~kW}$, therefore they have at least $2 \mathrm{~kW}$ solar power redundant which will be sold to other 30 nodes. As such, there are 25 selling and 30 buying prosumers, and the inter-prosumer communication graph has 750 edges. Initially, parameters $a_{i}$ and $b_{i}$ are randomly generated in the intervals $[0.005,0.009]$ and $[12.4,31.2]$, respectively.

Running the proposed decentralized ADMM approach for $\mathrm{P} 2 \mathrm{P}$ energy trading between prosumers gives us the results in Figure 11. As seen in Figure 11. the total selling and buying powers of prosumers 

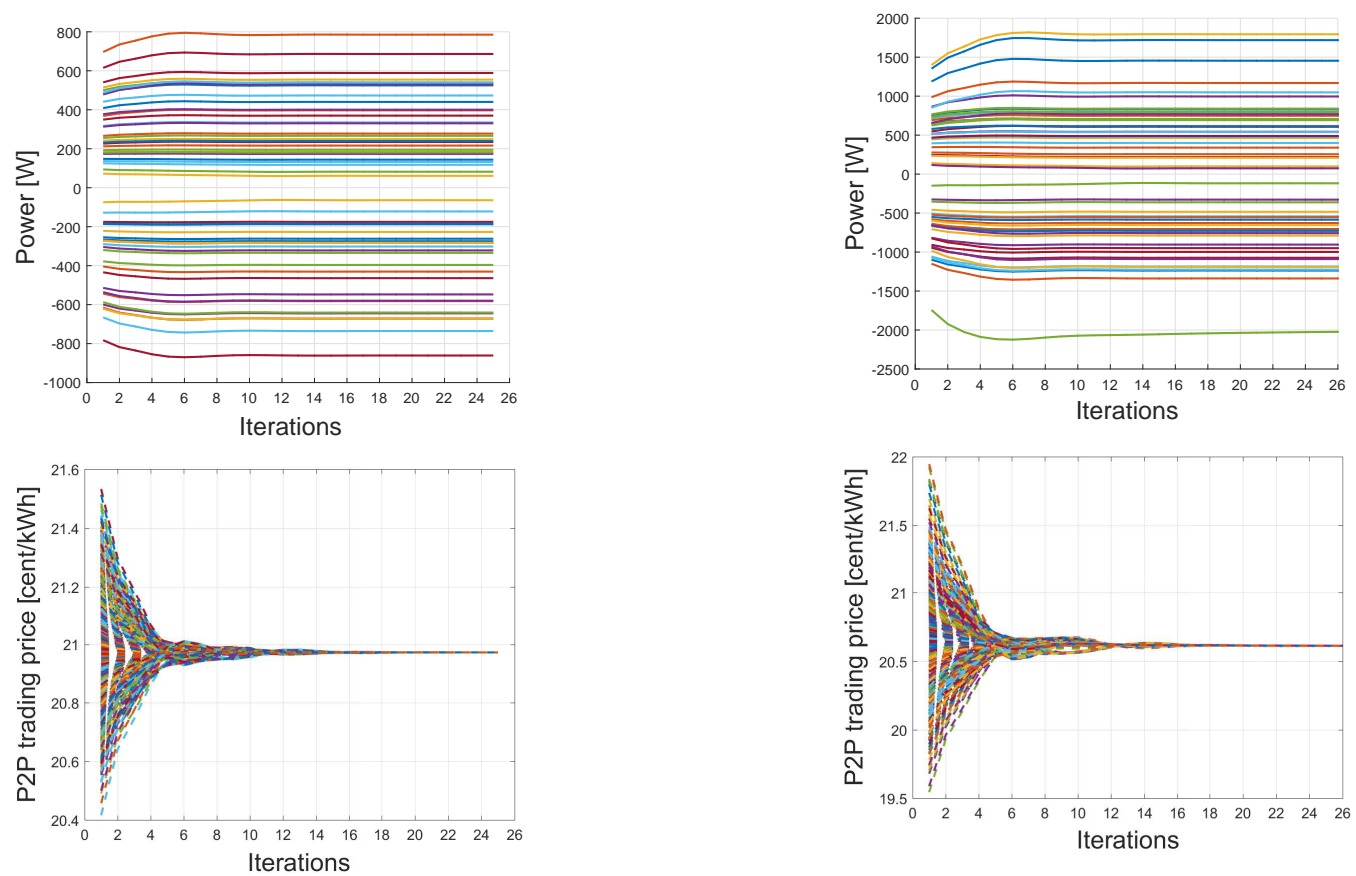

Figure 11: Convergence of total traded power and optimal price for $\mathrm{P} 2 \mathrm{P}$ trading between prosumers.

Figure 12: Convergence of total traded power and optimal price for prosumers $\mathrm{P} 2 \mathrm{P}$ trading when $a_{i}$ are decreased. 
are far from their maximum capacities. Thus, using the proposed decentralized learning method for tuning prosumers parameters, all prosumers reduces their parameters $a_{i}$ by randomly regenerating them in the interval $[0.002,0.006]$. The simulation results for the new values of $a_{i}$ are depicted in Figure 12. It is obviously observed in Figure 12 that the total traded power of prosumers are significantly increased in absolute values, which validates the proposed learning strategy. The optimal prices are not much different between two cases. Similar simulation results are obtained at other time steps.

The merits of P2P energy trading here are as follows. First, it reduces power flows from and to the bulk grid by local consumption, hence eases power losses and voltage/frequency problems. Second, households with renewable generation can save their investment costs by selling excessive renewable energy to other neighboring households instead of storing with battery storage systems.

\subsection{Scalability of The Proposed Ap- proach}

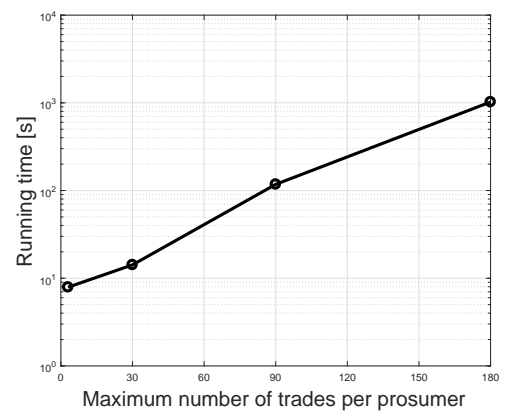

Figure 13: Computational time of the proposed distributed P2P ADMM approach vs. system size.

The proposed decentralized ADMM algorithm running times for the above two cases of IEEE European Low Voltage Test Feeder are 14.2s and 21.2s, which are just double of that for the synthetic system (c.f. Table 2), while the maximum number of communicated peers (i.e. trades) per prosumer is 30 , i.e. 10 times bigger. Next, the proposed algorithm is tested for larger systems, one with 75 selling and 90 buying prosumers, and the other with 150 selling and 180 buying prosumers. Running time for the former is $117.6 \mathrm{~s}$, while that for the latter is $1022.9 \mathrm{~s}$. As seen in Figure 13. computational time of the proposed P2P ADMM approach is not exponentially increased with system size, hence is scalable well.

Note that the computational time shown in Figure 13 does not take into account the inter-peer communication time which may contain delays. In practical implementation, communication latency is an issue needs to be carefully handled for both centralized, distributed, and decentralized approaches, due to the tradeoff between communication load and computational ability. Centralized and distributed approaches have the advantages of stronger computational capability at the central unit and less total number of communication links over decentralized approaches. On the other hand, all communication tasks occur at the central unit in centralized and distributed optimization approaches, of which communication latency could become a critical issue, while communication load at each agent in decentralized approaches could be much fewer. However, communication delay is out of scope of the current research, and will be addressed in the future work.

\section{Conclusion}

This paper has proposed a decentralized ADMM optimization approach for P2P energy markets and decentralized learning strategies for prosumers to obtain successful transactions and total traded powers as they expect. Analytical formulas for the amount of power and its associated transaction price in each trading between a pair of prosumers were derived, which reveal insights on the relation between individual trading with the total traded amount of each prosumer, trade weights, and prosumer cost function parameters. These serve as bases for the proposed decentralized learning strategies to tune prosumers cost function parameters, and for the choice of trade weights to attain strategic objectives such as pollutant emissions reduction. The effectiveness and scal- 
ability of the proposed approaches were illustrated through different case studies.

In the next research, more complex models of $\mathrm{P} 2 \mathrm{P}$ market participants, power flow constraints in the $\mathrm{P} 2 \mathrm{P}$ energy systems, and the interaction of $\mathrm{P} 2 \mathrm{P}$ markets with other markets should be investigated. Moreover, different factors such as communication delays, systematic way to choose trade weights, etc., should also be studied.

\section{Appendix}

\subsection{Proof of Theorem 1}

When $d_{i j}=0 \forall i, j=1, \ldots, n$, and the communication graph between successfully traded peers is connected, it is easy to see from (10) that $\lambda_{i j}^{*}$ are the same for all $j \in \mathcal{N}_{i}$, hence are the same for all $i=1, \ldots, n$ due to the symmetry of the trading and the connectedness of the communication graph. The unique price is calculated by $2 a_{i} P_{i, t r}^{*}+b_{i}=\lambda^{*}$. Based on this, we can easily derive $\lambda^{*}$ as in (11) due to the fact that $\sum_{i=1}^{n} P_{i, t r}^{*}=0$, and then obtain $P_{i, t r}^{*}$ as in (12).

Next, utilizing the KKT conditions to the social welfare maximization problem (6), we also obtain the clearing energy price equals to $\left.\frac{\partial C_{i}}{\partial P_{i, t r}}\right|_{P_{i, t r}^{*}}=$ $2 a_{i} P_{i, t r}^{*}+b_{i}$. As such, the energy price in this market is the same with that in $\mathrm{P} 2 \mathrm{P}$ market without trade weights. Consequently, it is obvious that the total traded energy of each peer in the $\mathrm{P} 2 \mathrm{P}$ market is equal to that in the pool-based market. In case this graph is unconnected, i.e. the considering $\mathrm{P} 2 \mathrm{P}$ market is clustered into smaller $\mathrm{P} 2 \mathrm{P}$ markets, each cluster inherits the above properties.

Finally, if $d_{i j} \neq 0$, then (10) can be stacked together to obtain $E^{T} P_{t r}^{*}=\operatorname{vec}\left(b_{j}+d_{j i}-b_{i}-d_{i j}\right)$. In addition, the condition $\sum_{i=1}^{n} P_{i, t r}^{*}=0$ is rewritten as $\alpha^{T} P_{t r}^{*}=0$. Therefore, we have (13).

\subsection{Proof of Theorem 2}

Substituting (27) into (26) gives us

$$
\begin{aligned}
& 2 a_{i} P_{i, t r}^{k+1}+(\rho+\phi) P_{i j}^{k+1}+v_{i j}^{k} \\
& =2 a_{j} P_{j, t r}^{k+1}-(\rho+\phi) P_{i j}^{k+1}+v_{j i}^{k}
\end{aligned}
$$

which is 28a . Using this to compute $\lambda_{i j}^{k+1}$ leads to (28b). Then summing up (28a) for all $j \in \mathcal{N}_{i}$ gives us

$P_{i, t r}^{k+1}=\frac{\sum_{j \in \mathcal{N}_{i}} v_{j i}^{k+1}+\sum_{j \in \mathcal{N}_{i}} 2 a_{j} P_{j, t r}^{k+1}-\sum_{j \in \mathcal{N}_{i}} v_{i j}^{k+1}-2 n_{i} a_{i} P_{i, t r}^{k+1}}{2(\rho+\phi)}$

which is equivalent to

$$
\begin{aligned}
& 2\left(\rho+\phi+n_{i} a_{i}\right) P_{i, t r}^{k+1}-\sum_{j \in \mathcal{N}_{i}} 2 a_{j} P_{j, t r}^{k+1} \\
& =\sum_{j \in \mathcal{N}_{i}} v_{j i}^{k+1}-\sum_{j \in \mathcal{N}_{i}} v_{i j}^{k+1}
\end{aligned}
$$

Equation (31) is rewritten exactly as (29). Next, the convergence of the proposed ADMM algorithm follows that provided in [7], hence we omit the proof here for brevity. Lastly, substituting the converged solutions to (26) leads to $\lambda_{i j}^{*}=2 a_{i} P_{i, t r}^{*}+b_{i}+d_{i j}+$ $\rho u_{i j}^{*}$. Thus, as $\rho \rightarrow 0, \lambda_{i j}^{*}=2 a_{i} P_{i, t r}^{*}+b_{i}+d_{i j}$, solutions in Theorem 1

\section{References}

[1] CVX: Matlab software for disciplined convex programming.

[2] P. Baez-Gonzalez, E. Rodriguez-Diaz, J. C. Vasquez, and J. M. Guerrero. Peer-to-Peer Energy Market for Community Microgrids. IEEE Electrification Magazine, 6(4):102-107, 2018.

[3] T. Baroche, F. Moretz, and P. Pinson. Prosumer markets: A unified formulation. In 2019 IEEE Milan PowerTech, pages 1-6, 2019.

[4] T. Baroche, P. Pinson, R. L.G. Latimier, and H. B. Ahmed. Exogenous Cost Allocation in Peer-to-Peer Electricity Markets. IEEE Transactions on Power Systems, 34(4):2553 - 2564, 2019. 
[5] S. Boyd, N. Parikh, E. Chu, B. Peleato, and J. Eckstein. Distributed optimization and statistical learning via the alternating direction method of multipliers. Foundations and Trends in Machine Learning, 3(1):1-122, 2011.

[6] H. Le Cadre, P. Jacquot, C. Wan, and C. Alasseur. Peer-to-peer electricity market analysis: From variational to Generalized Nash Equilibrium. European Journal of Operational Research, 282:753-771, 2020.

[7] W. Deng, M-J. Lai, Z. Peng, and W. Yin. Parallel multi-block admm with $o(1 / k)$ convergence. Journal of Scientific Computing, 71(2):712-736, 2017.

[8] European Commission. Photovoltatic geographical information system.

[9] J. Guerrero, A. C. Chapman, and G. Verbic. Decentralized P2P Energy Trading under Network Constraints in a Low-Voltage Network. IEEE Transactions on Smart Grid, 10(5):5163-5173, 2019.

[10] IEEE PES AMPS DSAS Test Feeder Working Group. IEEE Test Feeder Resources.

[11] J. Liu, S. Mou, and A. S. Morse. Asynchronous Distributed Algorithms for Solving Linear Algebraic Equationss. IEEE Transactions on Automatic Control, 63(2):372-385, 2018.

[12] N. Liu, X. Yu, W. Fan, C. Hu, T. Rui, Q. Chen, and J. Zhang. Online Energy Sharing for Nanogrid Clusters: A Lyapunov Optimization Approach. IEEE Transactions on Smart Grid, 9(5):34624-4636, 2018.

[13] N. Liu, X. Yu, C. Wang, C. Li, L. Ma, and J. Lei. Energy-Sharing Model With Price-Based Demand Response for Microgrids of Peer-to-Peer Prosumers. IEEE Transactions on Power Systems, 32(5):3569-3583, 2017.

[14] Yang Liu, C. Lageman, B. D.O. Anderson, and G. Shi. An Arrow-Hurwicz-Uzawa type flow as least squares solver for network linear equations. Automatica, 100:187-193, 2019.
[15] M. Khorasany and Y. Mishra and G. Ledwich. A Decentralised Bilateral Energy Trading System for Peer-to-Peer Electricity Markets. IEEE Transactions on Industrial Electronics, 67(6):4646-4657, 2019.

[16] F. Moret and P. Pinson. Energy Collectives: a Community and Fairness based Approach to Future Electricity Markets. IEEE Transactions on Power Systems. (Accepted). DOI: 10.1109/TPWRS.2018.2808961.

[17] T. Morstyn, N. Farrell, S. J. Darby, and M. D. McCulloch. Using peer-to-peer energy-trading platforms to incentivize prosumers to form federated power plants. Nature Energy, 3:94-101, 2018.

[18] T. Morstyn and M. D. McCulloch. Multi-Class Energy Management for Peer-to-Peer Energy Trading Driven by Prosumer Preferences. IEEE Transactions on Power Systems, 34(5):40054014, 2019.

[19] T. Morstyn, A. Teytelboym, and M. D. McCulloch. Bilateral Contract Networks for Peer-toPeer Energy Trading. IEEE Transactions on Smart Grid, 10(2):2026 - 2035, 2019.

[20] D. H. Nguyen, S. Azuma, and T. Sugie. Novel Control Approaches for Demand Response with Real-time Pricing using Parallel and Distributed Consensus-based ADMM. IEEE Transactions on Industrial Electronics, 66(10):7935$7945,2019$.

[21] D. H. Nguyen, T. Narikiyo, and M. Kawanishi. Optimal demand response and real-time pricing by a sequential distributed consensus-based ADMM approach. IEEE Transactions on Smart Grid, 63(6):1694-1700, 2018.

[22] Y. Riffonneau, S. Bacha, F. Barruel, and S. Ploix. Optimal Power Flow Management for Grid Connected PV Systems With Batteriess. IEEE Transactions on Sustainable Energy, 2(3):309-320, 2011. 
[23] E. Sorin, L. Bobo, and P. Pinson. Consensusbased approach to peer-to-peer Electricity Markets with Product Differentiation. IEEE Transactions on Power Systems, 34(2):994-1004, 2019.

[24] T.Sousa, T. Soares, P. Pinson, F. Moret, T. Baroche, and E. Sorin. Peer-to-peer and community-based markets: A comprehensive review. Renewable and Sustainable Energy Reviews, 104:367-378, 2019.

[25] W. Tushar, T. K. Saha, C. Yuen, T. Morstyn, M. D. McCulloch, H. V. Poor, and K. L.Wood. A motivational game-theoretic approach for peerto-peer energy trading in the smart grid. Applied Energy, 243:10-20, 2019.

[26] W. Tushar, C. Yuen, H. Mohsenian-Rad, T. Saha, H. V. Poor, and K. L. Wood. Peerto-Peer Trading in Electricity Networks: An Overview. IEEE Transactions on Smart Grid. (Accepted). DOI: 10.1109/TSG.2020.2969657.

[27] W. Tushar, C. Yuen, H. Mohsenian-Rad, T. Saha, H. V. Poor, and K. L. Wood. Transforming Energy Networks via Peer-to-Peer Energy Trading: The potential of game-theoretic approaches. IEEE Transactions on Power Systems, 35(4):90-111, 2018.

[28] A. Werth, A. Andre, D. Kawamoto, T. Morita, S. Tajima, M. Tokoro, D. Yanagidaira, and K. Tanaka. Peer-to-Peer Control System for DC Microgrids. IEEE Transactions on Smart Grid, $9(4): 3667-3675,2018$.

[29] K. Zhang, S. Troitzsch, S. Hanif, and T. Hamacher. Coordinated Market Design for Peer-to-Peer Energy Trade and Ancillary Services in Distribution Grids. IEEE Transactions on Smart Grid. (Early Access). DOI: 10.1109/TSG.2020.2966216. 